\title{
Design and analysis of an UFLS scheme for low-inertia based power grid
}

\author{
S. S. Hossain, M.J Hossain, and Edstan Fernandez \\ School of Engineering \\ Macquarie University \\ Sydney, NSW 2109, Australia \\ s-k.hossain@students.mq.edu.au \\ jahangir.hossain@mq.edu.au \\ edstan.fernandez@hdr.mq.edu.au
}

\author{
Md Shihanur Rahman \\ Engineer- National Planning \\ Operational Analysis \& Engineering \\ Australian Energy Market Operator \\ Shihanur.rahman@aemo.com.au
}

\begin{abstract}
A Significant change to power systems' dynamic behavior, especially frequency responses, following a contingency event is a major concern due to the high penetrations of low/inertia-less renewable energy sources. Power system inertia can be getting weaker with the integrations of renewable energy into the grid. As a result, sometimes the under frequency load shedding (UFLS) schemes fail to protect the frequency decline below the threshold limits with conventional settings. This paper addresses this problem and analyse the impacts of penetration of renewable energies into the power systems. Furthermore, a modified load-shedding method is proposed by considering the rate of change of frequency (ROCOF) and the total system's damping factor. Then a comparison study between proposed method and other methods (conventional and MILP) is presented. A 13-bus real power system is considered as test bus and several case studies are conducted using the Python based PSS/E simulation software platform. From the simulation results it is found that, the proposed load shedding method successfully restricts the frequency decline within a safe limits and thereby, avoids the possibility of major blackouts.
\end{abstract}

Index Terms-Blackouts, Under Frequency Load Shedding (UFLS), System Frequency Response (SFR), Rate of Change of Frequency (ROCOF), solar power plants, synchronous generators

\section{INTRODUCTION}

Power system blackouts have become one of the major concerns in recent years as they have an enormous impact on the socio-economic welfare. Due to the integration of large-scale intermittent converter-interfaced renewable energy sources, e.g. photovoltaic (PV), double-fed induction generator (DFIG), wind turbine etc., the current system's dynamic characteristics have started to change significantly. Though power-system stability has been recognized as an important problem since the 1920 s, recently it has drawn wider attraction due to recent changes in the dynamic behaviors of power systems. Due to the replacement of existing large generators by wind turbines, Photovoltaic units, and other renewable energy sources, the inertia of the system is reduced significantly, which contributed to increase the rate of change of frequency (ROCOF). As a result, probability of losing stability and reliability of the power grid is getting higher and more unpredictable in recent years.

Power systems usually operate around their stability limits, focusing more on economic objectives for operation. An unstable condition following a credible contingency event can lead cascaded tripping of generators, which might cause a major blackout in the system. In order to avoid the cascade tripping of generators, study of a system's frequency responses and adopting necessary schemes accordingly is mandatory to gain frequency stability under various contingencies. Frequency stability can be defined by the ability of a power system to continue at a stable frequency following several contingencies [1]. It can be achieved by matching the total generations and loads, with minimum unintentional loss of loads. There are two techniques commonly used to maintain grid stability- i) one method is to design a controller for DG sources (PV or Wind plant) or conventional generators in a way that they will operate at their normal power rating and provide the maximum power, when the system needs excess power to maintain grid stability $[2,3]$ and ii) the other one is to use under-frequency load shedding (UFLS) schemes.

UFLS is the most adopted and widely used technique to avoid the frequency declination after a disturbance. When any power imbalances occur between load and generation due to a sudden increase of load or generation shortage, the UFLS scheme can bring the frequency back into a safe operation limit by disconnecting possible minimum number of loads. The UFLS scheme is a conventional load shedding scheme where the amount of power deficit is not considered in load shedding design. Rather it is designed for the worst possible contingencies which may fail to protect the frequency stability with the addition of more renewable energy sources into the system. In order to overcome the aforementioned problem, an adaptive UFLS scheme comes into play to estimate the appropriate amount of load-shedding by using the frequency derivatives as a measure of the power deficit amount. In [4] an adaptive UFLS technique is proposed for an islanding distribution network which exhibits a frequency overshoot at the end of the frequency excursion process due to improper load shedding. In $[5,6]$ a new 
method of under- frequency load shedding is presented where the power deficit amount is considered but only the nadir frequency is focused upon. The steady-state response is necessary to evaluate the performance of that scheme. An optimized UFLS model is proposed following the optimization of the system frequency response (SFR) model for a large power system in [7]. In [8] a smart integrated adaptive centralized control method is proposed to minimize the UFLS by using the rate of change of frequency (ROCOF) measured from the Phasor Measurement Unit (PMU). Although the above methods provide some interesting results, none of them considered the impact of a changing frequency response due to the addition of renewable energy in the grid. Moreover in [9], a load shedding scheme is proposed where the power deficit amount and the impact of renewable energy, especially uncertainty of wind power generations is considered to solve the given problem. However, the frequency response of the grid with the allowable maximum power deviation amounts are not investigated.

By addressing the impact of high penetration of renewable energy on the conventional UFLS schemes, this paper proposes an improved load-shedding scheme. The proposed scheme considers worst-case scenario, which minimizes the amount of load shedding and eliminates the frequency-overshoot problem. In addition, a conventional UFLS scheme is developed and implemented in the test network and the deficiency of conventional load shedding scheme in the presence of renewable energy while arresting the frequency decline has also been addressed. The proposed load-shedding scheme is tested in a 13-bus real power network, which is a portion of the Bangladesh power grid network. From results it is found that the proposed method ensure stable frequency in the presence of low inertia based renewable energy. The rest of the paper is organized as follows: Section II describes the modeling of the proposed system; Section III presents a description of the test system; in Section IV case studies are presented, and finally Section $\mathrm{V}$ discusses the results followed by a conclusion in Section VI.

\section{MODELING OF PROPOSED SYSTEM}

The proposed UFLS scheme is designed by utilizing the formulation of swing equation. To design load shedding scheme properly, the amount of shortage of power or the power deficit, is required to be measured. The amount of power deficiency can be measured in two different ways. One is by detecting the status of breaker nearest to the generator and if the breaker is open, the most recent power output of that generator can be used as a measure signal. The other method is to calculate the deficit of power by using the swing equation [9]. In a renewable-source-connected grid system, the power may be shorted due various reasons, such as sudden loss of synchronous machines, lack of wind power, lack of sun light etc. In this paper, the second method is considered to measure the amount of deficiency of the power output. In order to determine the amount of load to be shed, the center-of-inertia frequency [9] can be calculated as:

$$
\mathrm{f}_{\mathrm{COI}}=\frac{\sum_{i=1}^{\mathrm{N}} \mathrm{H}_{\mathrm{i}} \mathrm{f}_{\mathrm{i}}}{\sum_{\mathrm{i}=1}^{\mathrm{N}} \mathrm{H}_{\mathrm{i}}}
$$

where $\mathrm{N}$ is the number of connected generators; $H_{i}$ is the inertia constant of each generator in seconds, and $f_{i}$ the frequency of each generators in Hertz.

The amount of load to be shed can be minimized by utilizing the spinning reserve of the system, which depends on the generators' capacity. The total spinning reserve can be calculated by:

$$
\mathrm{TSR}=\sum_{\mathrm{i}=1}^{\mathrm{N}} \mathrm{MGC}_{\mathrm{i}}-\sum_{\mathrm{i}=1}^{\mathrm{N}} \mathrm{AGP}_{\mathrm{i}}
$$

Where $N$ is the number of connected generator, $M G C_{i}$ the maximum capacity of the $i$ th generator, and $A G P_{i}$ the actual generated power of the $i$ th generator.

Then power deficit amount can be calculated by:

$$
\mathbf{P}_{\text {deficit }}=\frac{2 \times\left.\right|^{\mathrm{df}} \mathrm{COI}_{\mathrm{Cdt}} / \times \mathrm{H}_{\mathrm{sq}}}{\mathbf{f}_{\mathrm{m}}}
$$

Where $P_{\text {deficit }}$ is the power deficit; $d f_{\text {COI }} / d t$ the rate of change in center-of-inertia frequency deviation; $f_{n}$ is the nominal frequency which is $50 \mathrm{~Hz}$ in our case, and finally $H_{e q}$ is found by

$$
\mathrm{H}_{\mathrm{eq}}=\sum_{\mathrm{i}} \frac{\mathrm{H}_{\mathrm{i}} \mathrm{S}_{\mathrm{i}}}{\mathrm{s}}
$$

In other way, the deficiency of the output power can be estimated by using the information of disconnected sources as:

$$
\mathrm{P}_{\text {deficit }}=\sum_{=1}^{\mathrm{N}} \mathrm{P}_{\text {disconnected source }}
$$

Where $P_{d \& \text { ficit }}$ is the power imbalance and $N$ the number of disconnected source and $P_{\text {disconnected sourcs }}$ is the disconnected power of the generators. Then actual amount of load shedding can be evaluated as:

$$
\text { TLSA }=\mathbf{P}_{\text {deficit }}{ }^{-T S R}
$$

The primary frequency control can be started at frequency level $49.8 \mathrm{~Hz}[10]$. But it can be varied depending on the level of required protection. In Bangladesh, the lack of spinning reserve was one of the main reasons for the September 2014 blackouts. Therefore in the proposed loadshedding scheme, the spinning reserve is considered as zero (i.e.TSR $=0$ ). The impact of load damping (D) is also considered, which can reduce the load shedding amount. When an imbalance of power occurs due to disturbance, the amount of power deficiency is calculated from equation (3). Since the zero spinning reserve is considered in this work, so the total load shedding would be equal to the power deficit following (6). However in the proposed UFLS scheme, the system's total damping impact is considered, and will be calculated by the following equations [11]: 


$$
\beta=\mathrm{D}+\frac{1}{\mathrm{R}_{\mathrm{eq}}}
$$

where $\beta$ is the total damping factor; $D$ is the load-frequency sensitivity factor (value 2 is considered here); and $R_{e q}$ is the equivalent speed governor droop which can be calculated by:

$$
\frac{1}{\mathrm{R}_{\mathrm{eg}}}=\sum_{\mathrm{i}} \frac{1}{\mathrm{R}_{\mathrm{i}}}
$$

where $R_{i}$ is the speed droop constant of $i$ generators. In the proposed load shedding method, the maximum allowable frequency deviations is considered $\pm 0.5 \mathrm{~Hz}$. By taking this limit into consideration the formula to calculate the total amount of load shedding can be revised. Therefore, the total allowable power deficit amount can be calculated as:

$$
\Delta \mathrm{P}_{\max }=\beta \times \Delta \mathrm{f}_{3 \mathrm{~B}}
$$

where $\Delta f_{s s}=\frac{\text { maximum allowable frequency deviation }(0.5 \mathrm{~Hz})}{\text { Nominal frequency }(50 \mathrm{~Hz} \text { for our system) }} ; \Delta P_{\max }$ is the amount of maximum allowable power deficit; $\beta$ is the total system damping factor. Now the total amount of load shedding will be:

$$
\text { TLSA }=\mathrm{P}_{\text {deficit }}-\Delta \mathrm{P}_{\max }
$$

After any imbalance of power or any disturbance in the power system by using (3) the power deficit is calculated by using the breaker status of synchronous generators and center-of-inertia frequency. By considering the total system damping factor the necessary amount of load shed is calculated and the amount of load shedding in each of the 3 stages is distributed according to [6]. It is worth mentioning that, by taking the total system damping factor into account the amount of actual load shed can be minimized.

\section{TEST SYSTEM MODEL}

In this paper, a small portion from the Bangladesh power network is considered to implement the proposed UFLS scheme. This system consists of 7 synchronous generators (G1-G7) and 5 loads. The detail parameter of the system can be seen in [16]. The single line diagram is shown in Fig. 1. The total load on the system is, $\mathrm{P}_{\mathrm{L}}=434.46 \mathrm{MW}$ and $\mathrm{Q}_{\mathrm{L}}=149$ MVAR and the total generations capacity is, $\mathrm{P}_{\mathrm{G}}=590 \mathrm{MW}$. Transformer and the transmission lines is included in the reduced admittance matrix [17]. The generators are equipped with slow excitation system (IEEE-DC 1A) [14] and the loads are considered as static loads. In order to perform dynamic simulations and according to the recommendation of [13], constant current and constant impedance models are considered for the active and reactive components of static load modelling. Generators G1, G4, and G6 are replaced by constant power solar model to perform the dynamic simulations in the presence of renewable energy. The electrical models of the PV system with their associated control systems in PSS/E are taken from [15].

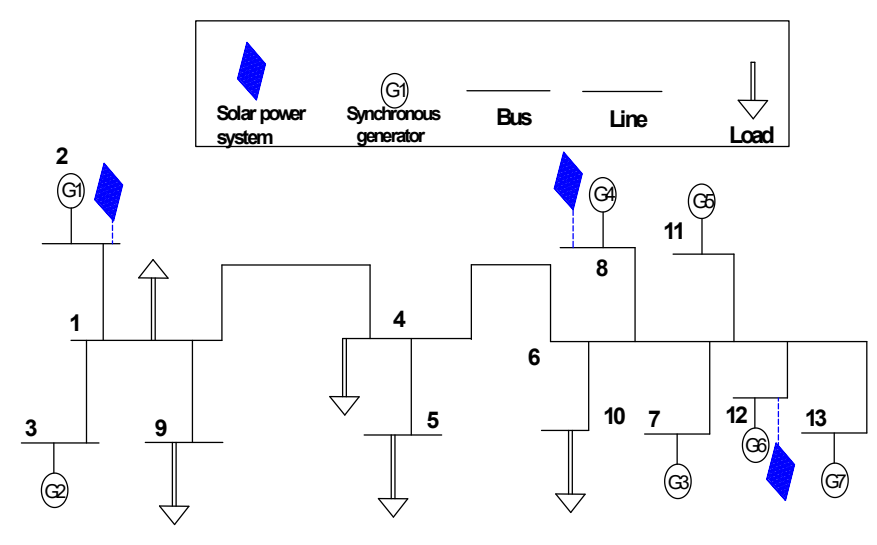

Fig. 1 Test Network

\section{SIMULATION RESULTS}

In a conventional UFLS settings the load shed amount is set out by considering the worst contingency condition or $50 \%$ of generation outage. According to IEEE guidelines for UFLS, load shedding can be done in 3 to 5 stages. Distribution of load shedding blocks among those steps can be performed in a manner like smaller blocks of load can be shed in earlier stage and increase the size of blocks with the increment of load shedding stages. In order to avoid more load shedding in the middle of contingencies, the total amount of load shedding is distributed into three blocks in which smaller blocks are kept in the primary stages. The above process is used to set the conventional UFLS parameters and the summary of the settings are given in Table 1:

\section{TABLE I CONVENTIONAL SETTINGS FOR UNDER FREQUENCY RELAY}

\begin{tabular}{|c|c|c|}
\hline $\begin{array}{c}\text { Frequency } \\
\text { (Hz) }\end{array}$ & Load shed (in P.U) & Time (Sec) \\
\hline 49.4 & 0.14 & 0.2 \\
\hline 48.6 & 0.16 & 0.2 \\
\hline 47.9 & 0.19 & 0.2 \\
\hline
\end{tabular}

The frequency excursion with the conventional settings without any renewable penetration is shown in Fig. 2.

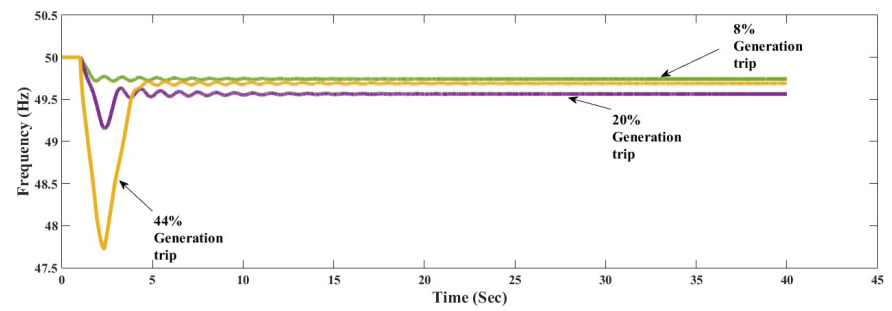

Fig. 2 Frequency responses before renewable energy penetration with conventional setting

However, with the integration of $27 \%$ of renewable energy sources, the conventional load shedding scheme exhibits the following frequency responses for different contingencies shown in the Fig. 3. 


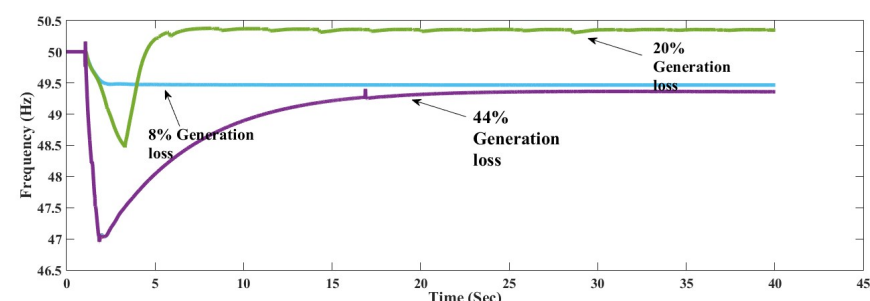

Fig. 3 Frequency responses after $27 \%$ renewable energy (Solar energy) penetration with conventional setting

The proposed UFLS scheme re-assessed the amount of load shedding and the traditional scheme is redesigned. The following case studies are carried out in order to see the impact of load shedding on the frequency excursion process and to observe the performance of the proposed UFLS scheme.

Case-1: Outage of $44 \%$ of generations in an islanded network

In this case study, the highest possible generation losses are considered. The conventional load shedding scheme failed to arrest frequency within the threshold limit $(47.5 \mathrm{~Hz})$ in the presence of renewable energy sources as shown in Fig. 4. This case study is performed to investigate whether the proposed load shedding can successfully arrest frequency excursion within the threshold limit or not. In this case, the test network is modelled with $27 \%$ of solar energy sources as constant power model. After the load flow study the frequency responses following a generation loss $(44 \%$ of total generation) at bus-13 is considered. In order to distribute the amount of load shedding, the procedure described in [9] is taken into account. In this process, the frequency deviations and the amount of load to be shed is calculated through microprocessor-based controller (which needs to be programmed). The controller sends the load shedding signals to the relay according to load priorities to disconnect the load. For UFLS functioning the assumption is made that the load shedding control action will be done from one point. The overall time would take around $200 \mathrm{~ms}$ [9] for the relay pickup time and time required for assessing the amount of load to be shed by sending signal to the breakers to disconnect that load. The modified UFLS settings and frequency response are shown in Table-II and Fig. 4.

TABLE II PROPOSED SETTINGS FOR UNDER FREQUENCY RELAY FOR 44\% OF GENERATION LOSS

\begin{tabular}{|c|c|c|}
\hline Frequency (Hz) & Load shed (in P.U) & Time (Sec) \\
\hline 49.4 & 0.21 & 0.2 \\
\hline 48.6 & 0.17 & 0.2 \\
\hline 47.9 & 0.09 & 0.2 \\
\hline
\end{tabular}

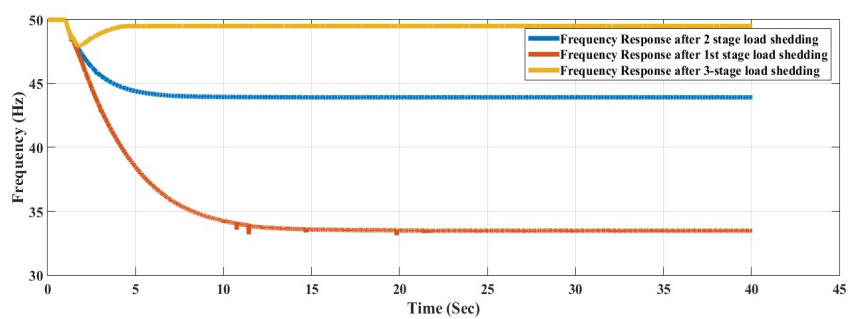

Fig. 4 Frequency responses of different stages of load shedding after $27 \%$ renewable energy (Solar energy) penetration with the proposed setting for $1^{\text {st }}$ contingency

Case-2: Outage of $20 \%$ of generations in an islanded network

The performance of the proposed load shedding scheme is tested for a middle contingency. In this scenario, the generators from bus -3 and bus- 7 are disconnected and the performance is evaluated. The modified UFLS settings and the frequency responses are shown in Table-IIIII and Fig. 5

TABLE III PROPOSED SETTINGS FOR UNDER FREQUENCY RELAY FOR 20\% OF GENERATION LOSS

\begin{tabular}{|c|c|c|}
\hline $\begin{array}{c}\text { Frequency } \\
(\mathbf{H z})\end{array}$ & Load shed (in P.U) & Time (Sec) \\
\hline 49.4 & 0.07 & 0.2 \\
\hline 48.6 & 0.05 & 0.2 \\
\hline 47.9 & 0.03 & 0.2 \\
\hline
\end{tabular}

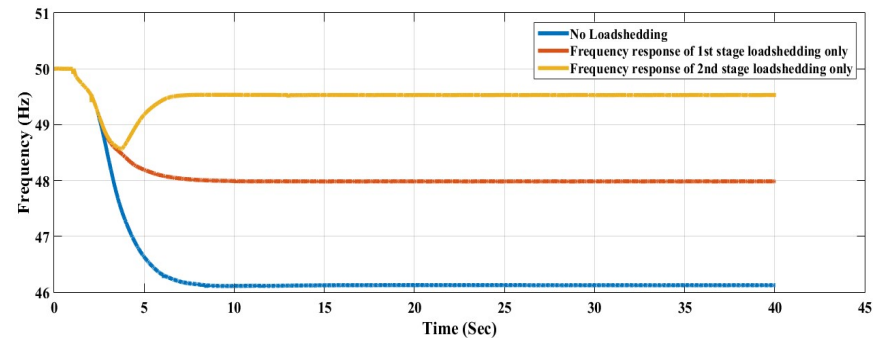

Fig. 5 Frequency responses of different stages of load shedding after $27 \%$ renewable energy (Solar energy) penetration with the proposed setting for 2nd contingency

Case-3: Outage of $8 \%$ of generations in an islanded network

In this case study, no load shedding is required by the proposed UFLS scheme at all as the frequency deviations are within the maximum limit due to the lowest contingency. Fig. 6 shows the frequency response due to $8 \%$ of generation loss

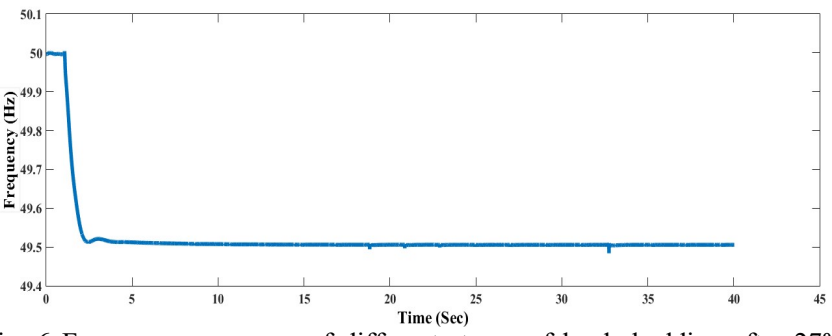

Fig. 6 Frequency responses of different stages of load shedding after $27 \%$ renewable energy (Solar energy) penetration with the proposed setting for 3rd contingency 


\section{RESULTS \& DISCUSSION}

The safety operating limit for the synchronous generators is $50.5-49.5 \mathrm{~Hz}$ for a $50 \mathrm{~Hz}$ power system [12]. Although it varies according to manufacturer's specifications, in most cases, synchronous machines will instantly trip at $47.5 \mathrm{~Hz}$. Therefore, the frequency excursion process should not allow the frequency below that level to avoid the synchronous generators to trip, which might lead to possible blackouts. With the integration of renewable energy sources into the grid the ROCOF becomes more sensitive and responsive to the disturbances. It is observed that, the UFLS with conventional settings suitably works for distributed synchronous generators, but it fails to arrest frequency declination when the large-scale renewable energy sources are integrated into the grid. Moreover, the proposed UFLS method has also been compared with another offline based UFLS method described in [18]. In Table- IV, the comparison of the proposed settings with the conventional settings and another proposed UFLS setting(MILP based) has been summarized for different contingencies $(44 \%, 20 \%$ and $8 \%$ of gen loss) based on the simulation results. Then in Fig. 7,8 and 9 comparison studies of frequency excursion process of conventional method, UFLS method proposed in [18] and the proposed on-line based UFLS method (considering load damping factor) have been shown for 3 contingencies.

TABLE IV COMPARISON BETWEEN THE PROPOSED AND CONVENTIONAL (CONV.) /MILP UFLS SETTINGS

\begin{tabular}{|c|c|c|c|c|c|c|}
\hline & Conv./MILP & Proposed & Conv./MILP & Proposed & Conv./MILP & Proposed \\
\hline Gen. loss (\%) & 44 & 44 & 20 & 20 & 8 & 8 \\
\hline $\begin{array}{c}\text { Nadir Freq. } \\
\text { (Hz) }\end{array}$ & $46.91 / 47.72$ & 47.88 & $48.48 / 48.70$ & 48.56 & $49.51 / 49.51$ & 49.51 \\
\hline $\begin{array}{c}\text { Steady state } \\
\text { Freq. (Hz) }\end{array}$ & $49.43 / 49.89$ & 49.5 & $50.42 / 49.63$ & 49.52 & $49.51 / 49.51$ & 49.51 \\
\hline $\begin{array}{c}\text { Load shed } \\
\text { (MW;MVAR) }\end{array}$ & $\begin{array}{c}64.79 / 215.03 ; \\
76.03\end{array}$ & $221 ; 86$ & $\begin{array}{c}64.79 / 77 ; \\
29.79\end{array}$ & $64 ; 27$ & None & None \\
\hline
\end{tabular}

From Table- IV, it is observed that the conventional UFLS settings can easily arrest frequency declination without any renewable energy penetration. On the other hand, although the conventional UFLS settings successfully arrest frequency for $20 \%$ and $8 \%$ generation loss, they fail to arrest frequency for $44 \%$ generation loss even with $27 \%$ penetration of renewable energy. The nadir frequency falls below $47.5 \mathrm{~Hz}$ which may lead to a cascade tripping of remaining synchronous generators. This causes more imbalance between power generation and consumption. The ROCOF becomes more sever in such condition which may lead to possible blackouts. On the other hand MILP based UFLS scheme also succeeded to restrict frequency declination beyond threshold limits. But for middle contingency it curtails more load than the proposed load shedding scheme. Moreover, an off-line based load shedding scheme has predefined load curtailment strategy which may fail to restrict frequency decline due to the uncertainty nature of loads. Whereas, in the proposed UFLS scheme load shedding amount and feeder to be shed are decided by the instant power deflection, which will try to avoid access or less load shedding and successfully bring back the frequency within the desired state. Hence, the proposed load shedding scheme provides excellent outcomes for the given contingency conditions which allow the power system to survive from possible major blackouts. In fact, the proposed load shedding scheme is applicable to any contingency that make the power system to be more robust to survive against any major blackouts. It is also worth to mention that for middle contingencies the proposed load shedding scheme exerted less amount of load shedding than the conventional settings. But in case of higher contingencies ( $44 \%$ gen. loss), due to the steeper response of ROCOF the proposed method executes more amount of load shedding though it is successful to restrict frequency falling within $47.5 \mathrm{~Hz}$ and thereby, avoiding blackouts.

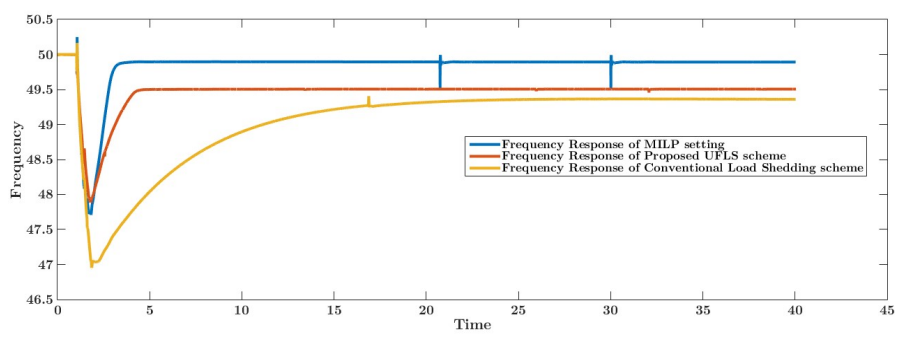

Fig.7 Frequency responses following $44 \%$ of generation loss

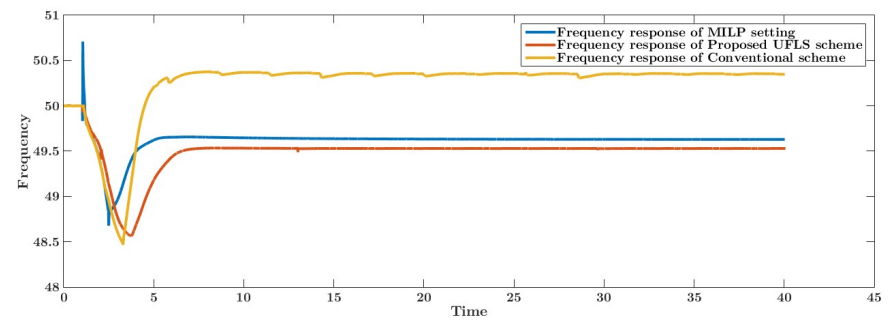

Fig. 8 Frequency responses following $20 \%$ of generation loss

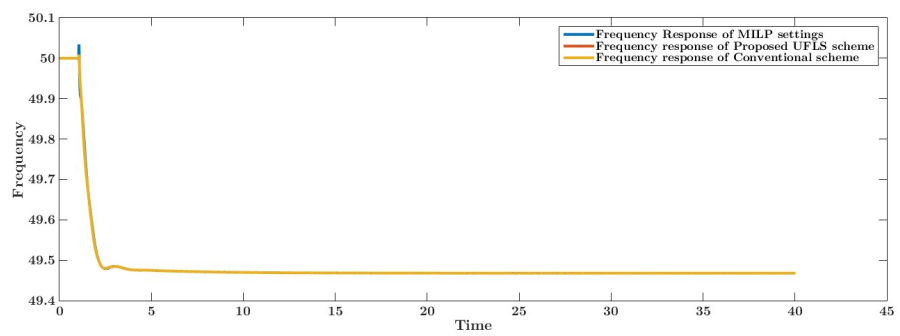

Fig.9 Frequency responses following $8 \%$ of generation loss 


\section{CONCLUSION}

The proposed UFLS scheme is based on the detection of the power deficit from the ROCOF. In order to minimize the amount of load shedding and to put the steady state frequency within the acceptable limits the total system damping factor is considered to calculate the amount of load shedding. Using the ROCOF to determine the load-shedding amount is one of the methods of calculating the amount of actual power imbalance. The conventional UFLS scheme, MILP based UFLS method and the proposed UFLS scheme are applied to a test network. The simulations are carried out by using Python based PSS/E simulator. The deficiencies of the conventional UFLS scheme are analyzed and a modified UFLS scheme is proposed to overcome the shortcomings of the problems related with the conventional scheme. Moreover, the frequency response and amount of load shedding are compared with the proposed UFLS scheme to evaluate the performance. In this paper, load shedding are carried out randomly, however, a priority based optimized load-shedding scheme may provide better performance.

\section{REFERENCES}

[1] Mohammad Ghaderi Darebaghi, Turaj Amraee: 'Dynamic multi-stage under frequency load shedding considering uncertainty of generation loss', IET Gener. Transm. Distrib., 2017, vol. 11 iss. 13, pp. 3202-3209

[2] Xin, H., Liu, Y., Wang, Z., et al.: 'A new frequency regulation strategy for photovoltaic systems without energy storage', IEEE Trans. Sustain. Energy, 2013, 4, (4), pp. 985-993

[3] Mauricio, J.M., Marano, A., Gómez-Expósito, A., et al.: 'Frequency regulation contribution through variable-speed wind energy conversion systems', IEE Trans. Power Syst., 2009, 24, (1), pp. 173-180

[4] Jung, J., Liu, C.-C., Tanimoto, S.L., et al.: 'Adaptation in load shedding under vulnerable operating conditions', IEEE Trans. Power Syst., 2002, 17, (4), pp.1199-1205.

[5] Ketabi, A., Fini, M.H.: 'An underfrequency load shedding scheme for hybrid and multiarea power systems', IEEE Trans. Smart Grid, 2015, 6, (1), pp. 82-91.
[6] Ketabi, A., Fini, M.H.: 'An underfrequency load shedding scheme for islanded microgrids', Int. J. Electr. Power, 2014, 62, pp. 599-607

[7] Deyou Yang, Shiyu Liu and Guowei Cai: 'Optimal System Frequency Response Model and UFLS Schemes for a Small Reciving-End Power System after Islanding', Appl. Sci. 2017, 7,

468; doi: 10.3390/app7050468, 'http: //www.mdpi.com/journal/applsci'.

[8] M. Karimi, R. Azizipanah-Abarghooee, H. Uppal, Q. Hong, C. Booth, and V. Terzija: 'Smart Integrated Adaptive Centralized Controller for Islanded Microgrids under Minimized Load Shedding', $20175^{\text {th }}$ International Istanbul Smart Grid and Cities Congress and Fair (ICSG), Year: 2017, pp.: $41-45$

[9] Jafar Jallad, Saad Mekhilef, Hazlie Mokhlis, Javed Ahmad Laghari, "Improved UFLS with consideration of power deficit during shedding process and flexible load selection" IET Renew. Power generation, 2018 , vol. 12 , Iss. 5 , pp. $565-575$

[10] Karimi, M., Mohamad, H., Mokhlis, H., et al.: 'Under-frequency load shedding scheme for islanded distribution network connected with mini hydro', Int. J. Electr. Power Energy Syst., 2012, 42, (1), pp. 127138

[11] P. //kundur, et al., Power system stability and control. New York: Mcgraw-Hill, 1994

[12] "IEEE Guiide for Abnormal Frequenc Protection for Power Generating Plants,"IEEE Std C37.106-2003 (Revision of ANSI/IEEE C37.1061987), pp.0_1-34, 2004.

[13] IEEE Task Force, "Load representation for dynamic performance analysis," IEEE Trans. Power Syst., vol. 8, no. 2, pp. 472-482, May1993.

[14] B. Pal and B. Chaudhuri, Robust Control in Power Systems. New York: Springer, 2005

[15] X. Wang and M. Yue, "Design of Energy Storage System to Improve Inertial Response for Large Scale PV Generation," 2016 IEEE Power and Energy Society General Meeting (PESGM), 17-21 July 2016, Electronic ISSN: 1944-9933

[16] https://pgcb.org.bd/PGCB/user/grid-network.php

[17] M. J. Hossain, Hemanshu R. Pota, Md. Apel Mahmud, and Rodrigo A.Ramos, "Investigation of the Impacts of Large-Scale Wind Power Penetration on the Angle and Voltage Stability of Power Systems" IEEE Systems Journal, vol. 6, no. 1, March

[18] F. Ceja-Gomez, S. S. Qadri, and F. D. Galiana, "Under-Frequency Load Shedding Via Integer Programming," IEEE Trans. Power Syst., vol. 27, no. 3, pp. 1387-1394, Aug. 2012. 